University of Wisconsin Milwaukee UWM Digital Commons

Theses and Dissertations

December 2016

\title{
If or When? Uncertainty's Role in Anxious Anticipation
}

Kenneth P. Bennett

University of Wisconsin-Milwaukee

Follow this and additional works at: https://dc.uwm.edu/etd

Part of the Biological Psychology Commons

\section{Recommended Citation}

Bennett, Kenneth P., "If or When? Uncertainty's Role in Anxious Anticipation" (2016). Theses and Dissertations. 1350.

https://dc.uwm.edu/etd/1350

This Thesis is brought to you for free and open access by UWM Digital Commons. It has been accepted for inclusion in Theses and Dissertations by an authorized administrator of UWM Digital Commons. For more information, please contact open-access@uwm.edu. 


\section{IF OR WHEN? UNCERTAINY'S ROLE IN ANXIOUS ANTICIPATION}

by

Kenneth Bennett

A Thesis Submitted in

Partial Fulfillment of the

Requirements for the Degree of

Master of Science

in Psychology

at

The University of Wisconsin - Milwaukee

December 2016 


\begin{abstract}
IF OR WHEN? UNCERTAINY'S ROLE IN ANXIOUS ANTICIPATION
\end{abstract}

by

\author{
Kenneth Bennett
}

The University of Wisconsin-Milwaukee, 2016

Under the Supervision of Professor Christine L. Larson

Uncertainty is often associated with subjective distress and a potentiated anxiety response. Occurrence uncertainty (OU), or the inability to predict if a threat will occur, has never been compared experimentally with temporal uncertainty (TU), or the inability to predict when a threat will occur. The current study aimed to 1) directly compare the eye-blink startle responsivity of $\mathrm{OU}$ and $\mathrm{TU}, 2$ ) develop a more effective task for isolating uncertain anticipation, and 3) better understand the relationship between individual differences in the intolerance of uncertainty and uncertain anticipation startle responsivity. The novel study showed that OU anticipation is more anxiety provoking than certain threat, but TU anticipation is superior at eliciting anxiety overall. This suggests that in the context of uncertainty, there must be some level of certainty or threat proximity to elicit a robust anxiety response. Individual difference in the intolerance of uncertainty were not related to anticipatory startle responsivity. 
(C) Copyright by Kenneth Bennett, 2016

All Rights Reserved 
To

my mother, my brother,

and my late father - thank you for your unlimited support. 


\section{TABLE OF CONTENTS}

PAGE

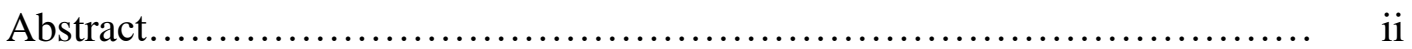

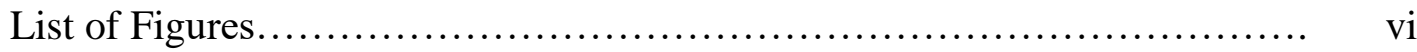

List of Tables......................................................... vii

I. Introduction....................................................... 1

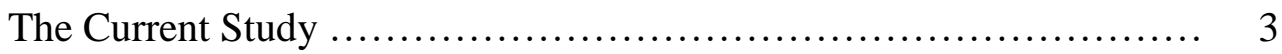

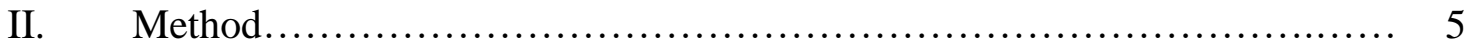

Participants.................................................. 5

Study Flow .................................................. 5

Materials and Procedures......................................... 6

Shock Work-Up....................................... 6

Startle Habituation...................................... 6

Occurrence Uncertainty vs. Temporal Unpredictability Task..... 7

Eye-blink Startle Collection................................ 8

Subjective Ratings.................................... 9

Assaying Intolerance of Uncertainty...................... 9

III. Analysis Plan...................................................... 9

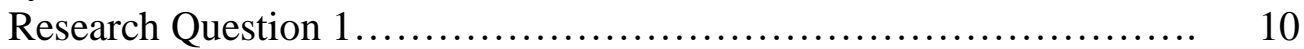

Research Question 2.......................................... 10

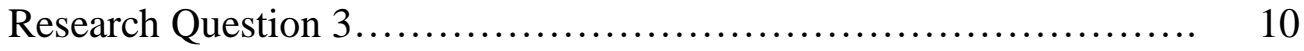

IV. $\quad$ Results......................................................... 11

Eyeblink Startle Magnitude: Anticipation Period.................... 11

Eyeblink Startle Magnitude: Inter-trial Intervals.................... 11

Anxiety Induction Manipulation Check............................ 11

Relations Between Anticipation Period Startle Responding and

Intolerance of Uncertainty Scores.................................. 12

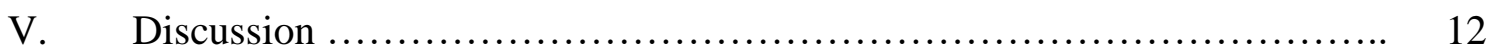

Disentangling Temporal and Occurrence Uncertainty................ 13

Individual Differences in Intolerance to Uncertainty and Anticipation

of Uncertain Threat................................................ 14

Carry-over of Anxiety......................................... 15

Conclusion..................................................... 15

VI. Figures \& Tables ............................................. 17

VII. References ................................................... 23 


\section{LIST OF FIGURES}

Figure 1. Anticipation Period Loading Bars................................ 17

Figure 2. Startle Blink Magnitudes During the Anticipation Period ................. 18

Figure 3. Startle Blink Magnitudes During the ITI period ...................... 19

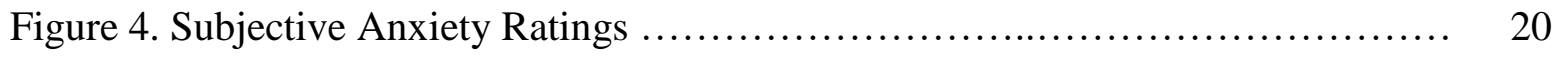

Figure 5. Shock-related Pain Ratings .................................... 21 


\section{LIST OF TABLES}

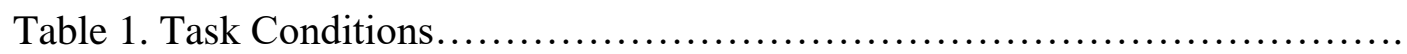




\section{ACKNOWLEDGEMENTS}

I want to thank my advisor, Christine Larson, for her continued support in my development as a student, researcher, and clinician. She has provided me with many unique research opportunities and always challenges me to accomplish my research and career goals. I am extremely grateful for her immense knowledge, wisdom, and caring support. I thank Jacqueline Dickmann for her interest in this research project and her diligent work to help get it started. Finally, I would like to thank my fellow lab mates (current and former) - Kevin Haworth, Tara Miskovich, Walker Pedersen, Ashley Huggins, Daniel Stout, and Emily Belleau. You have offered helpful perspectives and have provided me with the necessary tools and confidence to pursue my research interests.

The Advanced Opportunities Program Fellowship from UW-Milwaukee's Graduate School was also immensely helpful. This fellowship provided me with the substantial time and reduced workload to complete this manuscript. I am very grateful for this opportunity. 


\section{If or When? Uncertainty's Role in Anxious Anticipation}

Uncertainty is the inability to determine a situation's outcome or to predict the valence, intensity, occurrence, or type of future stimulus (Grupe \& Nitschke, 2013). Typically, anticipating uncertain potential threat and adjusting cognitively (e.g., planning out a means of response) and physiologically (e.g., fight or flight response) can be beneficial for the individual, especially when the threat is imminent and likely to occur (Grupe \& Nitschke, 2013). In contrast, extreme anxiety is thought to be developed and maintained by excessive anticipatory processes in the face of uncertainty (Grupe \& Nitschke, 2013). For example, panic attacks that occur unpredictably tend to substantially increase worry about if and when a panic attack will occur, leading to chronic anxious apprehension (Craske, Glover, \& DeCola, 1995). The tendency of anxious individuals to maladaptively anticipate uncertain threat or negative outcomes has received substantial attention, with mounting evidence suggesting that uncertainty is associated with heightened physiological reactivity and increased recruitment of brain regions that support the expression of arousal and negative affect, such as fronto-limbic circuits (Grillon, Baas, Lissek, Smith, \& Milstein, 2004; Grillon et al., 2006; Grillon et al., 2008; Grupe \& Nitschke, 2013; Nelson \& Shankman, 2011; Sarinopoulos et al., 2010; Shankman et al., 2014; Williams et al., 2014).

One of the most common paradigms used to measure response to uncertainty is the NPU task, which compares threat/fear responses in temporally predictable threat $(\mathrm{P})$, temporally unpredictable threat (U) and no threat (N) conditions (Grillon et al., 2004). Typically, the defensive startle blink response is enhanced during the unpredictable conditions, labeled anxietypotentiated startle, and during the predictable threat conditions, known as fear-potentiated startle (Schmitz \& Grillon, 2012). Results from studies examining the response to uncertain threat in 
non-anxious subjects using the NPU paradigm have revealed an increase in startle response when the aversive event was temporally uncertain compared to when the aversive event was certain and to safe conditions. The NPU paradigm has also effectively identified heightened response to uncertainty in various anxiety-related disorders, including panic disorder, generalized anxiety disorder, posttraumatic stress disorder, and childhood anxiety disorders (Grillon et al., 2008; Grillon et al., 2009; Williams et al., 2014). Thus, the NPU paradigm appears to be a useful tool for measuring response to uncertainty in both anxious and non-anxious populations, with recent evidence indicating that the NPU task has good psychometric properties (Kaye, Bradford, \& Curtin, 2016).

Although there has been a significant amount of research examining uncertainty, there is still a need to better characterize the various types of uncertainty. The majority of studies that have measured uncertainty with a task manipulation, such as the NPU task, have manipulated temporal uncertainty (TU), or the inability to predict when an aversive stimulus will occur (Grillon et al., 2004; Grillon, 2008; Grillon et al., 2008; Grillon et al., 2009; Lissek et al., 2005; Nelson \& Shankman, 2011; Shankman, Robison-Andrew, Nelson, Altman, \& Campbell, 2011). In contrast, relatively little is known about other aspects of uncertain threat, such as uncertainty regarding the intensity or likelihood of the threat occurring. A handful of studies have experimentally manipulated the intensity and frequency of threat (Bradford, Magruder, Korhumel, \& Curtin, 2014; Chin, Nelson, Jackson, \& Hajcak, 2015; Shankman et al., 2011). Shankman and colleagues (2011) found that temporal uncertainty produced significantly larger startle responses than conditions in which the intensity of the aversive stimulus was uncertain, highlighting the affective impact of temporally uncertain threat. Focusing on the uncertainty of the frequency or likelihood of the aversive stimulus, Chin and colleagues (2015) found greater 
startle magnitudes during high (75\%) reinforcement or more certain, compared to low (50\%) reinforcement conditions in an associative learning study designed to assess response to uncertainty. However, self-reported intolerance of uncertainty was positively correlated with increased startle blink magnitudes only during the $50 \%$ reinforcement, or more uncertain condition. The type of uncertainty elicited by this condition is not knowing if a threat will occur, suggesting that individuals who are less tolerant of uncertainty become more anxious when the occurrence of the potential threat is less certain.

The findings from Chin and colleagues (2015) highlight the possible relevance of occurrence uncertainty (OU), the inability to determine if (i.e., the likelihood) an aversive stimulus will occur, for eliciting anxious states (Bradford et al., 2014; Chin et al., 2015; Dunsmoor, Bandettini, \& Knight, 2007; Dunsmoor, Bandettini, \& Knight, 2008; Hsu, Bhatt, Adolphs, Tranel, \& Camerer, 2005; Williams et al., 2014). Occurrence uncertainty has been rarely studied in humans; therefore, it is unclear the state of anticipatory anxiety this type of uncertainty elicits, nor what role it plays in the development of chronic and clinical anxiety (Fujii, Uchida, \& Imada, 1994; Marlin, Sullivan, Berk, \& Miller, 1979). Although TU and OU are similar in the underlying assumption that threat is more salient when it is not predictable, it is not yet known if TU and OU are equally anxiety-provoking and there are no studies to our knowledge that have compared both experimentally. Because of uncertainty's potential role in the development of anxiety and the lack of clarity regarding what types of uncertainty are most anxiety provoking there is a clear need to address this significant gap in the literature.

\section{The Current Study}

The current study directly compared anxiety elicited by temporal and occurrence uncertainty by measuring the magnitude of the startle blink response while participants were 
under threat of potential shock in four anticipation conditions: 1) TU, or uncertainty as to when a potential threat would occur, 2) OU, or uncertainty as to if a potential threat would occur, 3) certain (C) threat, or absolute certainty a threat would occur at a given time, and 4) safe (S), in which no aversive stimulus occurred. We predicted that startle blink during the anticipation period would be significantly potentiated during uncertainty conditions (occurrence and temporal) compared to predictable threat and safe conditions, and that startle would be significantly larger during the anticipation period for certain threat compared to safe trials (Grillon et al., 2004; Grillon et al., 2006; Grillon et al., 2008; Nelson \& Shankman, 2011). Based on findings from Chin and colleagues (2015) that more certain (75\% reinforcement) threat elicited larger startle responses compared to less certain (50\% reinforcement) threat, we predicted that TU would elicit more anticipatory anxiety (e.g., larger startle blinks) than OU. However, given the paucity of research on occurrence uncertainty, this was an exploratory hypothesis. We also measured relationships between anticipatory anxiety during OU and TU with self-reported intolerance of uncertainty.

In addition to our primary research question, comparing the anxiogenic effects of TU and OU, we also used this study as an opportunity to alter the standard NPU task to isolate the anticipation and inter-trial interval (ITI) periods to more precisely separate anticipation of certain or uncertain threat from potential carry-over effects. In most studies using the NPU paradigm the aversive stimulus is explicitly unpaired from cue stimuli and can occur at any time, whereas during predictable threat the aversive stimulus will never occur during the ITI (Grillon, 2002; Grillon et al., 2004; Grillon et al., 2008; Nelson \& Shankman, 2011; Shankman et al., 2011). The task design used in the current study clearly separates anticipation periods from the ITI; the aversive stimulus only occurred at the end of an anticipation period and never during an ITI. This 
allowed us to test anxiety elicited both by anticipation of uncertain threat and whether that anxiety carries over into the safe ITI periods in these conditions. Lastly, we also examined relations between startle blink response to the four conditions and self-reported intolerance of uncertainty.

\section{Method}

\section{Participants}

Fifty-one undergraduates from the University of Wisconsin-Milwaukee completed the study for course extra credit and a \$15 Amazon gift card. Participants were at least 18 years old, proficient in English, and had no visual or hearing impairments (corrected vision was acceptable). The final sample consisted of 42 participants (30 female; $\left.M_{\text {age }}=20.8, S D_{\text {age }}=0.71\right)$. Three participants were dropped due to data collection errors, 3 due to processing errors, and 3 were classified as non-responders for the startle eyeblink (Bradford, Starr, Shackman, \& Curtin, 2015). Prior to the start of the experiment, participants provided written informed consent. Prior to conducting the study, a power analysis was conducted to determine the required sample size. Assuming a small to moderate effect size (partial $\mathrm{n}^{2}=0.37$ ) from Nelson and Shankman's study (2011), a power of 0.95 and alpha of 0.05 yielded a required sample size of 28 . Therefore, more than 50 participants were recruited to account for incomplete data, subject withdrawal, and dropped data due to artifact rejection.

\section{Study Flow}

Prior to the start of the task, participants underwent a shock threshold work-up to determine the level of electrical stimulation to be used throughout the task and completed a startle habituation procedure. Next, participants completed two runs of four blocks of the certainuncertain threat task. Each block contained trials from one condition: Certainty (C); Occurrence 
Uncertainty (OU); Temporal Uncertainty (TU); or Safe (S). The magnitude of physiological defensive responding was used as an index of anxiety and was measured as the magnitude of blink responses to acoustic startle probes during the anticipation and ITI periods. At the end of each block, participants rated their level of anxiety and discomfort associated with the shocks. After finishing the task participants completed a questionnaire assessing intolerance of uncertainty.

\section{Materials and Procedure}

Shock Work-Up. Participants first completed a shock work-up to set the level of electrical stimulation (i.e., a shock) at an individually-titrated painful, but tolerable, level. Shocks were delivered using Psychlab's SHK1 Pain Stimulation Shocker (Contact Precision Instruments, Cambridge, MA). Participants had two sensors placed approximately two inches above their right ankle (using double sided tape and conductive gel), which delivered the electric stimulation. Participants were told that they would receive a mild electric shock and would be asked to rate it from 1 to 10,1 being "didn't feel anything," and 10 being "painful, but tolerable." The goal was to work up to a level that the participant would subjectively rate as a 10: "painful, but tolerable." Once that shock level was established, shock was set at that level for the duration of task; the participant could increase or decrease the level at any point in the study if they became too uncomfortable. One participant increased their shock level from 100 to 120 units $(1.96 \mathrm{~mA}$ to $2.35 \mathrm{~mA})$ during the break in between runs and their data was included in analyses. All other participants maintained their initial shock level.

Startle Habituation. Once the shock work-up was completed, participants then began a startle habituation procedure. Following past research, this process was used to habituate the participants to the startle probes to prevent biased (excessively large) startle responses in the first 
few trials of the task (Blumenthal et al., 2005). Two sensors were applied under the left eye to measure the startle eye-blink response. This response was measured while participants were presented nine 50ms 102dB white noise startles through Bose noise cancelling headphones. There was an 8-12 s ITI between each startle probe. Immediately after startle habituation participants began the task.

Occurrence Uncertainty vs. Temporal Unpredictability Task. To create an anticipation period prior to delivery of the electrical stimulation (or lack thereof), participants viewed "loading bars" that slowly filled over the course of the anticipation period. These loading bars and associated instructions specified whether they will or might be shocked (threat) during the trial, at the end of each trial (when the bar was completely full), or not at all. The task was blocked by condition, and the start of each trial featured a cue slide explicitly stating which of the four conditions the participant was currently completing. The condition cue was replaced by a fixation cross after the anticipation period ended. Each trial's anticipation period consisted of a loading bar that filled over the course of up to $10 \mathrm{~s}$. The four conditions measured occurrence uncertainty and temporal uncertainty: 1) $\mathrm{C}$, with a shock always occurring at the end of the loading bar (10s); 2) TU, with a shock always occurring at a random time between $2 \mathrm{~s}$ and $10 \mathrm{~s}$ during the loading bar; 3) OU, with 50\% chance of a shock occurring at the end of the loading bar (10s); and 4) S, with no shocks throughout the loading bar (10s).

The task included eight blocks, each of which contained five trials of a single condition, for a total of 40 trials, 10 per condition. Similar to the Schmitz \& Grillon (2012) NPU task, we used two counterbalanced run orders: TU-S-C-OU and OU-C-S-TU. There was a five-minute break in between the two runs. Each trial lasted for a maximum of 35 seconds and contained one startle probe during the loading bar anticipation period and one during the ITI, which varied 
between $9 \mathrm{~s}$ and $24.5 \mathrm{~s}$. There were a total of 12 startle probes per condition, six during the bar, and six during the ITI, for a total of 48 startle probes overall (Table 1). Each startle probe was timed in such a way that there would always be at least 10s between startle probes and at least 10s following the aversive event (i.e., shock) to avoid the possibility of participants desensitizing to the startle probe (Grillon et al., 2008). Additionally, there was one shock delivered in each of the TU and $\mathrm{C}$ trials, one shock delivered in $50 \%$ of the OU trials, and no shocks delivered during the $\mathrm{S}$ trials, for a maximum of 15 shocks per run and 25 shocks overall (Table 1).

Eye-blink Startle Collection. Raw startle blink peak amplitudes were the primary dependent variable, consistent with previous startle blink investigations (Bradford et al., 2015; Grillon et al., 2004; Grillon et al., 2009; Nelson \& Shankman, 2011; Nelson, Hajcak, \& Shankman, 2015). Startle eye-blink data were measured using a BioNomadix ${ }^{\circledR}$ 2Ch EMG Receiver (Biopac Systems, Goleta, CA) from two 4-mm Ag/AgCl sensors placed below the left eye, over the orbicularis muscle. One sensor was placed $1 \mathrm{~cm}$ below the pupil and the other was placed $1 \mathrm{~cm}$ towards the outer canthi of the left eye. The ground sensor was placed in the center of the forehead. Blinks were recorded and processed using Biopac's Acqknowledge software according to guidelines presented by Blumenthal and colleagues (2005). Startle EMG was filtered online using a 5-500 Hz bandpass filter and offline using a $28 \mathrm{~Hz}$ high-pass filter $\left(4^{\text {th }}\right.$ order Butterworth) and a $30 \mathrm{~Hz}$ low-pass filter (4 ${ }^{\text {th }}$ order Butterworth). Peak amplitudes were measured in the $20-200 \mathrm{~ms}$ time window following the white noise startle probe. Trials were rejected if there was greater than $\mathrm{a} \pm 40 \mu \mathrm{V}$ deflection in the $50 \mathrm{~ms}$ baseline period. Blinks were visually inspected and were rejected if the startle response did not begin and end within the 20200 ms time window. Participants were determined to be non-responders if mean startle reactivity was below $5 \mu \mathrm{V}$ (Bradford et al., 2015). 
Subjective Ratings. At the end of each block, participants completed short subjective ratings to gauge their emotions during the task. They were asked to rate on a scale of one to seven how anxious they felt during the preceding block of trials and how aversive the electrical stimulation felt during that block. These subjective ratings were used as a manipulation check to determine if the electrical stimulation was perceived as aversive, consistent with previous research using an NPU paradigm (Nelson \& Shankman, 2011).

Assaying Intolerance of Uncertainty. The Intolerance of Uncertainty Scale (Buhr \& Dugas, 2002; Freeston, Rhéaume, Letarte, Dugas, \& Ladouceur, 1994; Freeston et al., 1994) was used to measure participants' level of intolerance of uncertain threats, situations, and outcomes. Intolerance of uncertainty scores were correlated with startle blink responsivity to better examine the relationship between the $\mathrm{OU}$, and TU, and $\mathrm{C}$ conditions. The IUS consists of 27 items and four factors: 1) uncertainty leads to the inability to act; 2) uncertainty is stressful and upsetting; 3) unexpected events are negative and should be avoided; 4) being uncertain about the future is unfair. The IUS has good test-retest reliability at a five-week interval $(r=.74)$, excellent internal consistency $(\alpha=0.94)$, and good internal and external validity with measures of anxiety, depression, and worry (Buhr \& Dugas, 2002).

\section{Analysis Plan}

Research Question 1: Replicating past findings, startle blink magnitude during anticipation will be greater for uncertain than certain conditions, and greater for certain than safe trials.

The first research question addressed whether startle blink magnitudes during anticipation periods would replicate findings from past literature (Grillon et al., 2004; Grillon et al., 2006; Grillon et al., 2008; Nelson \& Shankman, 2011). My hypothesis was that startle during the anticipation period would be significantly potentiated during uncertain conditions (occurrence 
and temporal) compared to predictable and safe conditions. I also predicted that startle would be significantly larger during the anticipation period for certain threat compared to safe trials. A one-way repeated measures ANOVA was conducted to examine the main effect of condition for startle blink magnitudes during the anticipation period. Follow-up Bonferroni corrected comparisons were used to examine differences between conditions.

Research Question 2: Comparison of startle response for TU and OU

For the second research question I compared anticipation startle blink magnitudes for TU and OU trials. I predicted that the anticipatory startle responses during TU trials would be significantly greater than those during OU trials. Using the same one-way repeated measures ANOVA described above (TU, OU, C, S), I predicted that there would be a main effect of condition. I conducted follow-up comparisons to determine if startle responses during TU were greater than those during OU trials.

Research Question 3: Relationship between startle response and IUS scores

The third research question examined the relationship between IUS scores and the anticipation startle response during certain and uncertain trials. I predicted that the startle response during uncertain trials would be more strongly related to IUS scores than certain conditions. Separate correlations were run to examine the OU-IUS and TU-IUS relationships. If there had been any significant correlations, I would have run a step-wise linear regression to examine the relationship between anticipation startle response during certain and uncertain trials and IUS scores. There were no significant correlations so this test was not run. 


\section{Results}

\section{Eyeblink Startle Magnitude: Anticipation Period}

A within-subjects ANOVA was conducted to examine the effect of condition during the bar anticipation period. Results revealed a main effect of condition, $F(3,42)=16.34, p<.0001$. Pairwise comparisons (Bonferroni-corrected) indicated that blinks during the TU anticipation period were significantly larger than those for the OU, C, and S conditions, $p s<.0001$ (Figure 2). Startle blinks were also larger during the OU anticipation period compared to the $\mathrm{C}, p<.012$, but not $\mathrm{S}$ anticipation periods, $p=.44$. Startle eyeblink magnitudes did not differ for $\mathrm{C}$ and $\mathrm{S}$ anticipation periods, $p>.99$.

\section{Eyeblink Startle Magnitude: Inter-trial Intervals}

An analogous ANOVA was conducted for the ITI period to examine potential anxietyrelated carry-over effects of startle responsivity into the always safe inter-trial interval. There was a significant main effect of condition, $F(3,42)=3.10, p<.05$. Post-hoc tests indicated that startle magnitudes during the TU block ITIs were nearly, but not quite significantly higher than those during C block ITIs, $p=.051$ (Figure 3; Bonferroni-adjusted). No other conditions were different from each other after Bonferroni correction, $p \mathrm{~s}>.20$

\section{Anxiety Induction Manipulation Check}

A series of repeated measures ANOVAs were conducted to determine whether the shock manipulation did indeed influence self-reported anxiety and pain. For shock-related anxiety there was a main effect of condition, $F(3,42)=67.55, p<.0001$. Pairwise comparisons revealed that participants rated feeling more anxious in the TU and OU conditions than in the $\mathrm{C}$ condition, which in turn was rated as more anxiety provoking than the $\mathrm{S}$ condition, $p s<.001$ (Figure 4). Finally, results indicated a main effect of condition on shock-related pain, $F(3,42)=152.37, p<$ 
.0001. Participants reported significantly less shock-related pain in the S condition compared to all threat conditions, $p \mathrm{~s}<.0001$ (Figure 5). Again, there were no significant differences among the threat conditions, $p \mathrm{~s}>.18$.

\section{Relations Between Anticipation Period Startle Responding and Intolerance of Uncertainty Scores}

A series of pairwise Pearson correlations were run to examine the relationships between anticipation startle responsivity during each condition and IUS scores. There were no significant correlations between the intolerance of uncertainty and startle responsivity, $p s>.83$.

\section{Discussion}

A direct comparison of TU and OU provided evidence for TU's superiority in eliciting anxiety-potentiated startle responsivity in a non-clinical sample. The findings support the robust extant literature indicating that uncertainty associated with not knowing when an aversive event will occur (TU) is a potent elicitor of anxiety (Grillon et al., 2004; Grillon, 2008; Grillon et al., 2008; Grillon et al., 2009; Lissek et al., 2005; Nelson \& Shankman, 2011; Shankman et al., 2011). Our results further reinforce the anxiogenic properties of uncertainty more broadly. While OU-elicited startle responsivity was less than that for TU, it was significantly larger than the anticipation startle responsivity during the certain threat $(\mathrm{C})$ condition. Therefore, $\mathrm{OU}$, an understudied type of uncertainty, may still be an important aspect of uncertainty relevant for evoking anxiety. Additionally, somewhat unexpectedly we found that anticipatory startle during $\mathrm{C}$ and $\mathrm{S}$ conditions were not different. These findings were not anticipated and should be interpreted cautiously. Finally, we did not find strong evidence for carryover of anxiety after the end of the anticipation in any condition, nor did we find that startle-blink assessed anticipatory anxiety was associated with self-reported intolerance of uncertainty. 


\section{Disentangling Temporal and Occurrence Uncertainty}

Although OU and TU have not been directly compared previously and OU has received relatively little focus, Chin and colleagues (2015) provided evidence for OU's role in the elicitation of anxiety-potentiated startle. They examined frequency as a type of uncertainty in an associative learning task of response to uncertainty with two conditions: a more uncertain $50 \%$ reinforcement condition (similar to our OU condition) and a less uncertain $75 \%$ reinforcement condition. The $75 \%$ reinforcement condition elicited a larger anticipatory startle response than the $50 \%$ condition. Their results suggest that, having some certainty that a threat will occur, but not knowing when, is a stronger potentiator of anxiety than conditions in which there is uncertainty about whether the threat will occur. This is consistent with our finding of greater blink potentiation in the TU condition, where participants were $100 \%$ certain that they would be shocked, but they did not know when it would occur. The threat during the OU condition may not have been salient enough to elicit a significantly larger startle response when compared to a temporally uncertain threat that was more certain. Interestingly, participants' self-reported anxiety at the end of each block indicated that in retrospect it felt equally anxiety-provoking anticipating both types of uncertainty, but, the online assessment of anticipatory anxiety showed a stronger effect for temporal uncertainty. Overall, our data indicate that although being completely certain about if and when a threat will occur is less anxiety-provoking than uncertain threat, some certainty that threat will occur enhances anticipatory anxiety in uncertain conditions.

This suggests that the threatening stimulus may need to be salient or close enough temporally or spatially (i.e., more certain) to trigger a more potent anxiety response. Indeed, previous literature examining the time course of anticipatory anxiety showed that when the startle response is elicited during the threat anticipation period, threat potentiation is not evident 
until just prior to the onset of the threat; in other words, the anticipatory anxiety dramatically increases as the threat becomes more certain to occur. (Grillon, Ameli, Merikangas, Woods, \& Davis, 1993; Grillon, Ameli, Goddard, Woods, \& Davis, 1994). Animal research has also supported the assertion that a defensive response pattern is governed by the physical distance of the threat, such that anxiety is initially produced in the presence of distal threat, and significantly increases as the threat becomes more imminent (Blanchard, Yudko, Rodgers, \& Blanchard, 1993; Bolles \& Fanselow, 1980; Davis, Walker, Miles, \& Grillon, 2010; Fanselow, 1986). Without this threat imminence, merely introducing uncertainty may not substantially increase the anxiety-potentiated startle response in non-anxious humans. Overall, it seems that there is a need for some degree of certainty (e.g., likelihood, timing, type) about an uncertain threat for the anticipatory anxiety to be significantly potent.

\section{Individual Differences in Intolerance to Uncertainty and Anticipation of Uncertain Threat}

Individual differences in the intolerance of uncertainty may be relevant for the way an individual differentially responds to temporal uncertainty and occurrence uncertainty. However, the findings thus far are mixed. For example, in the Chin and colleagues (2015) study, intolerance of uncertainty was positively correlated with the more uncertain condition (OU), rather than the less uncertain condition. Therefore, the more intolerant a person is of uncertainty, the stronger their anxiety-potentiated startle response is to uncertainty, regardless of the imminence of the threat. However, in our study individual differences in the intolerance of uncertainty did not differentially affect how an individual physiologically responded to temporal versus occurrence uncertainty. One possible explanation for why intolerance of uncertainty was not correlated with anticipation startle responsivity for any of the conditions is that there may not have been a large enough sample to capture a broader sufficient range in intolerance of 
uncertainty scores. However, our sample's scores ranged from 31 to $96(M=63.31, S D=17.15)$, thus capturing a wide variability in intolerance of uncertainty. Another explanation is that intolerance of uncertainty is not associated with startle responsivity in this modified NPU task. The short, isolated anticipation periods may not provide enough time for any physiological nuances to emerge in the face of occurrence or temporal uncertainty. More research is needed to understand how individual differences in self-reported intolerance of uncertainty may influence differential anxious anticipation responsivity in the face of different types of uncertainty.

\section{Carry-over of Anxiety}

In a secondary analysis we did not find much evidence of the carryover effect of threat across conditions during the ITI period, when the task was safe. It was the case that startle responsivity during the $\mathrm{TU}$ condition was nearly significantly larger than that during the $\mathrm{C}$ condition; however, there were no other significant simple comparisons. Although there may have been a small carry-over effect for the TU condition (relative to $\mathrm{C}$ ), the overall findings suggest that our task isolated the ITI period from the anticipation and made it effectively safe. Fear-potentiated startle to uncertain threat therefore may only be transiently anxiety provoking once the individual either experiences the threat or knows they are in a safe environment, thus minimizing the potential for carry-over effects (Grillon et al., 1993; Mobbs et al., 2007;

Shankman et al., 2011).

\section{Conclusion}

The anticipation of uncertain threat is a strong elicitor of acute anxiety. Temporal and occurrence uncertainty both elicited greater anticipatory anxiety than certain threat, supporting the large body of research that uncertain threat tends to elicit significant anxiety (Bradford et al., 2014; Grillon et al., 2004; Grillon et al., 2008; Grupe \& Nitschke, 2013; Nelson \& Shankman, 
2011; Nelson et al., 2015; Schmitz \& Grillon, 2012; Shankman et al., 2011). Our results suggest that although the uncertainty regarding the occurrence of a threatening stimulus is enough to elicit anxiety, a temporally unpredictable, but certain-to-occur threat elicits more potent anticipatory anxiety. Therefore, threat that is certain to occur may be more relevant for eliciting an anxiety-potentiated startle response in the context of uncertainty. For example, prior to a job performance review a person would likely experience significant anticipatory anxiety if they knew that an aspect of their performance was poor (e.g., interpersonal skills with customers), but they did not know when in the review it would be addressed. More research is needed to further characterize the aspects of uncertainty that are most relevant for the experience of anxiety. Future research should focus on the anticipation period as a means for manipulating the expectation about a future stimulus, such as the length of the anticipation period, the time at which anxiety is measured (e.g., when the startle probe occurs with respect to the threat), or the impact of participant control over the degree of uncertainty of the threat.

Overall, the findings provide more information about uncertainty's role in the anticipation of threat and gives more support for its potential role in the development and maintenance of anxiety (Barlow, 2000; Foa, Zinbarg, \& Rothbaum, 1992; Mineka \& Kihlstrom, 1978). These findings also provide insight into how varying aspects of uncertainty may be related to anticipatory anxiety, and highlight the particularly potent anxiogenic impact of temporal uncertainty. 
Figure 1. Anticipation Period Loading Bars.

A.

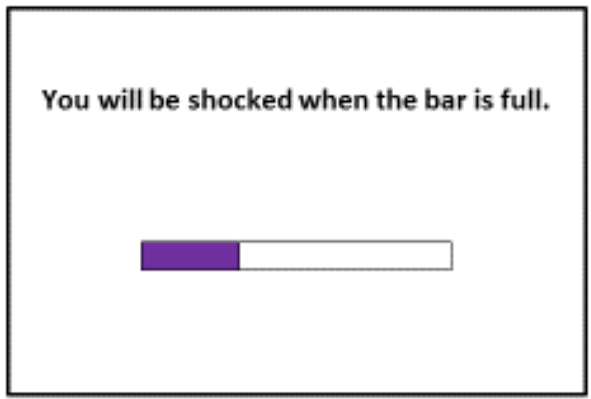

c.

You might be shocked when the bar is full.
B.

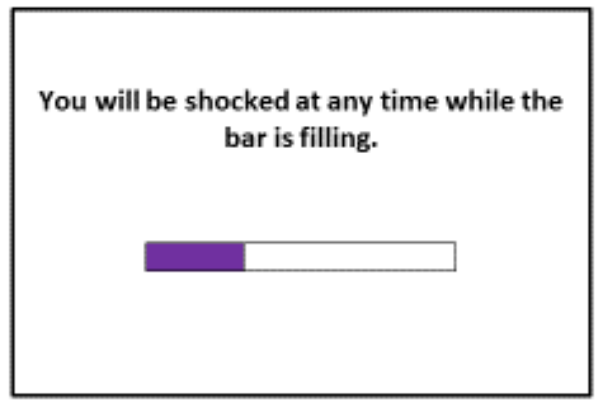

D.

You will not be shocked at any time.

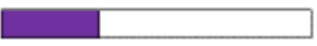

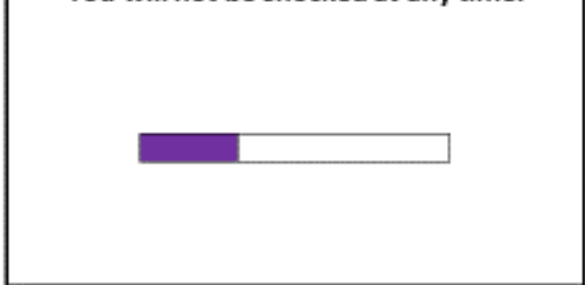

An example of the four task condition anticipation periods while "loading bars" are filling. A.) Certainty (C). Participants are shocked when the bar is completely filled (100\% purple). B.) Temporal Uncertainty (TU). Participants are shocked (100\%) but could occur at any time while the bar is filling. C.) Occurrence Uncertainty (OU). Participants may or may not be shocked (50\% shock rate) when the bar is completely filled. D.) Safe (S). Participants will not be shocked at any point during the trial, including the ITI. 
Figure 2. Startle Blink Magnitudes During the Anticipation Period

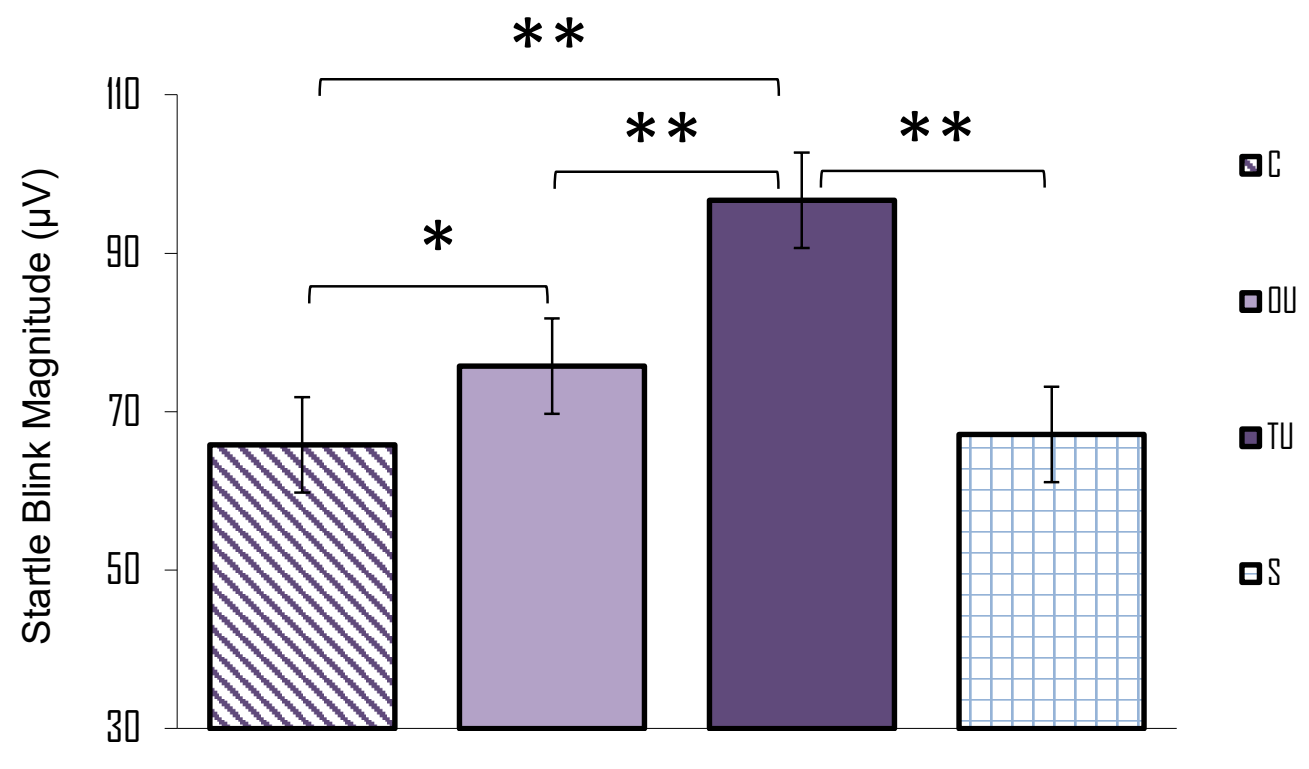

Startle blink magnitudes during the anticipation period (while "loading bars" are filling). From left to right: Certain (C), Occurrence Uncertainty (OU), Temporal Uncertainty (TU), and Safe (S). * $=p<.05$; ** $=p<.0001$. 
Figure 3. Startle Blink Magnitudes During the ITI Period

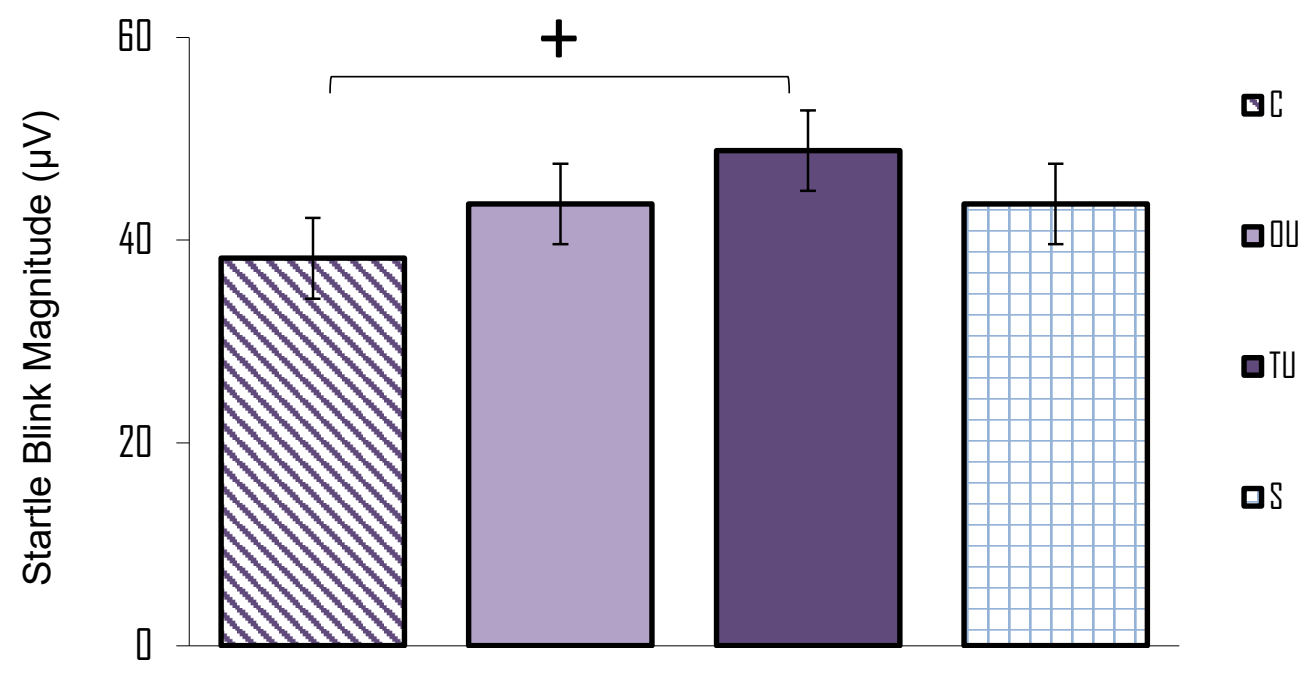

A bar graph representing Bonferonni-corrected follow-up comparisons after an ANOVA for the startle responsivity during the ITI period (i.e., while "loading bars" were not present and it was safe from threat). From left to right: Certain (C), Occurrence Uncertainty (OU), Temporal Uncertainty (TU), and Safe (S). ${ }^{+}$ $=p=.051$. 
Figure 4. Subject Anxiety Ratings

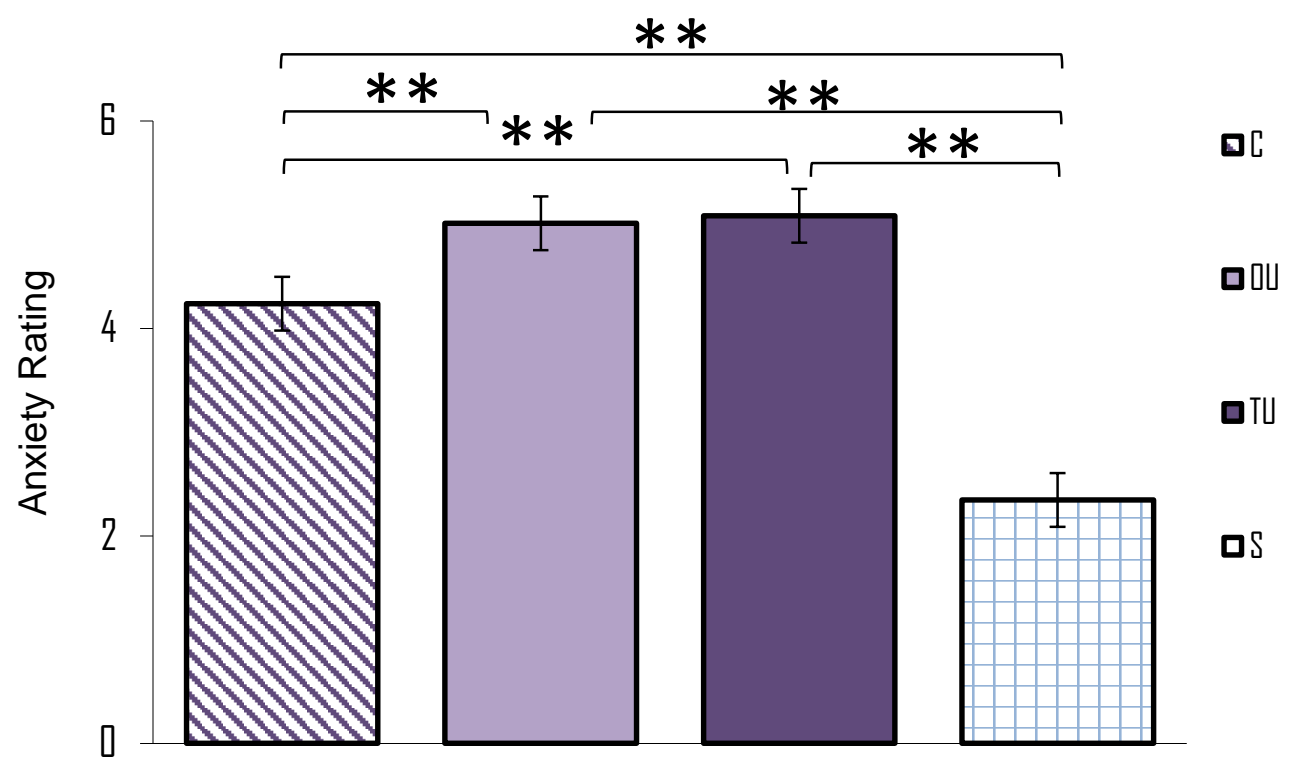

A bar graph representing Bonferonni-corrected follow-up comparisons after an ANOVA for the anxiety ratings recorded at the end of each block. From left to right: Certain $(\mathrm{C})$, Occurrence Uncertainty (OU), Temporal Uncertainty (TU), and Safe (S).** $=p<.0001$. 
Figure 5. Shock-related Pain Ratings

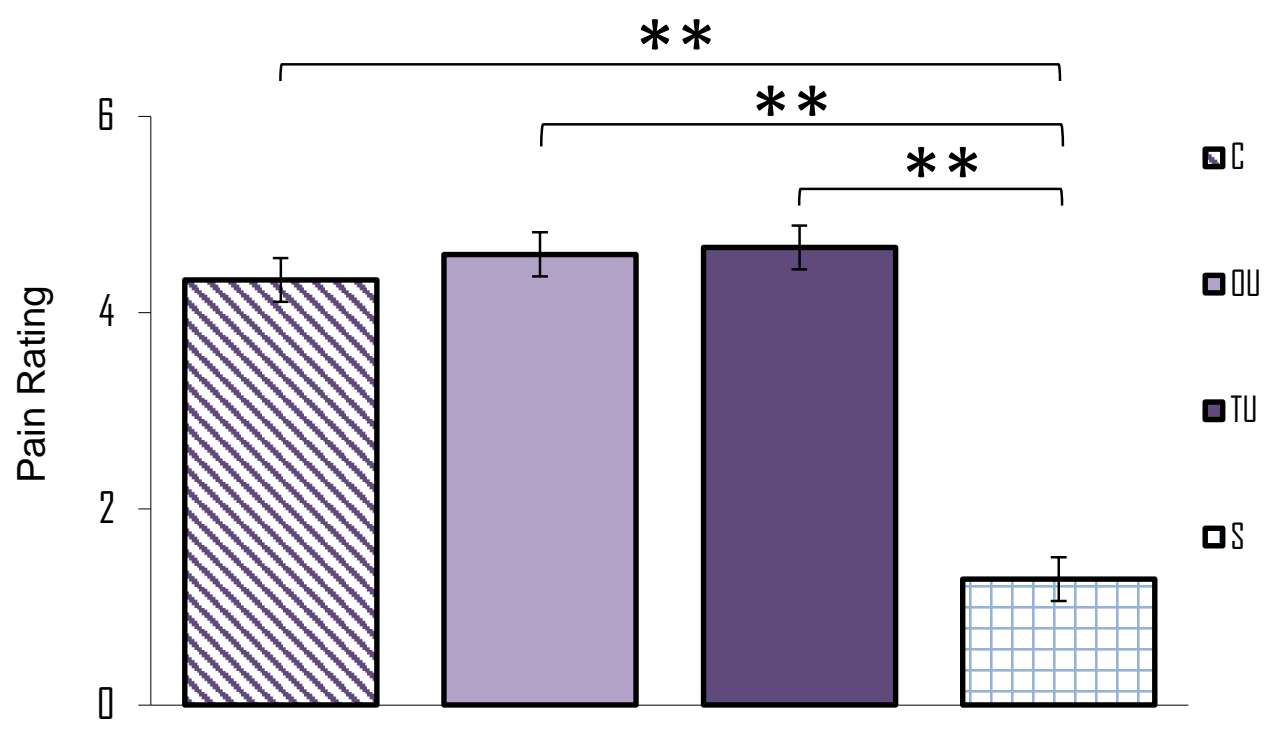

A bar graph representing Bonferonni-corrected follow-up comparisons after an ANOVA for the shock pain ratings recorded at the end of each block. From left to right: Certain $(C)$, Occurrence Uncertainty (OU), Temporal Uncertainty (TU), and Safe (S). ** $=p<.0001$. 
Table 1. Task Conditions

\begin{tabular}{lcccc}
\hline Condition & Bar Length & Trials & Shocks & Startles \\
\hline Certainty (C) & $10 \mathrm{~s}$ & 10 & 10 & 12 \\
Temporal Uncertainty (TU) & $2 \mathrm{~s}-10 \mathrm{~s}$ & 10 & 10 & 12 \\
Occurrence Uncertainty $(\mathbf{O U})$ & $10 \mathrm{~s}$ & 10 & 5 & 12 \\
Safe $(\mathbf{S})$ & $10 \mathrm{~s}$ & 10 & 0 & 12 \\
\hline Total & & 40 & 25 & 48 \\
\hline
\end{tabular}

The four task conditions and number of trials, shocks, and startle probes are listed: C: certain shock, certain time; TU: certain shock, uncertain time; OU: uncertain shock, certain time; and S: no shock (safe). 


\section{References}

Barlow, D. H. (2000). Unraveling the mysteries of anxiety and its disorders from the perspective of emotion theory. American Psychologist, 55(11), 1247.

Blanchard, R. J., Yudko, E. B., Rodgers, R. J., \& Blanchard, D. C. (1993). Defense system psychopharmacology: An ethological approach to the pharmacology of fear and anxiety. Behavioural Brain Research, 58(1), 155-165.

Blumenthal, T. D., Cuthbert, B. N., Filion, D. L., Hackley, S., Lipp, O. V., \& Van Boxtel, A. (2005). Committee report: Guidelines for human startle eyeblink electromyographic studies. Psychophysiology, 42(1), 1-15.

Bolles, R. C., \& Fanselow, M. S. (1980). A perceptual-defensive-recuperative model of fear and pain. Behavioral and Brain Sciences, 3(02), 291-301.

Bradford, D. E., Starr, M. J., Shackman, A. J., \& Curtin, J. J. (2015). Empirically based comparisons of the reliability and validity of common quantification approaches for eyeblink startle potentiation in humans. Psychophysiology, 52(12), 1669-1681.

Bradford, D. E., Magruder, K. P., Korhumel, R. A., \& Curtin, J. J. (2014). Using the threat probability task to assess anxiety and fear during uncertain and certain threat. Journal of Visualized Experiments: JoVE, (91), 51905-51905.

Buhr, K., \& Dugas, M. J. (2002). The intolerance of uncertainty scale: Psychometric properties of the english version. Behaviour Research and Therapy, 40(8), 931-945.

Chin, B., Nelson, B. D., Jackson, F., \& Hajcak, G. (2015). Intolerance of uncertainty and startle potentiation in in relation to different threat reinforcement rates. International Journal of Psychophysiology, 99, 79-84. 
Craske, M. G., Glover, D., \& DeCola, J. (1995). Predicted versus unpredicted panic attacks: Acute versus general distress. Journal of Abnormal Psychology, 104(1), 214.

Davis, M., Walker, D. L., Miles, L., \& Grillon, C. (2010). Phasic vs sustained fear in rats and humans: Role of the extended amygdala in fear vs anxiety. Neuropsychopharmacology, $35(1), 105-135$.

Dunsmoor, J. E., Bandettini, P. A., \& Knight, D. C. (2007). Impact of continuous versus intermittent CS-UCS pairing on human brain activation during pavlovian fear conditioning. Behavioral Neuroscience, 121(4), 635.

Dunsmoor, J. E., Bandettini, P. A., \& Knight, D. C. (2008). Neural correlates of unconditioned response diminution during pavlovian conditioning. NeuroImage, 40(2), 811-817.

Fanselow, M. S. (1986). Associative vs topographical accounts of the immediate shock-freezing deficit in rats: Implications for the response selection rules governing species-specific defensive reactions. Learning and Motivation, 17(1), 16-39.

Foa, E. B., Zinbarg, R., \& Rothbaum, B. O. (1992). Uncontrollability and unpredictability in post-traumatic stress disorder: An animal model. Psychological Bulletin, 112(2), 218.

Freeston, M. H., Rhéaume, J., Letarte, H., Dugas, M. J., \& Ladouceur, R. (1994). Why do people worry? Personality and Individual Differences, 17(6), 791-802.

Fujii, M., Uchida, M., \& Imada, H. (1994). Effects of presence and absence of information about shock intensity upon licking suppression in rats. Japanese Psychological Research, 36(2), $65-73$.

Grillon, C. (2002). Startle reactivity and anxiety disorders: Aversive conditioning, context, and neurobiology. Biological Psychiatry, 52(10), 958-975. 
Grillon, C. (2008). Greater sustained anxiety but not phasic fear in women compared to men. Emotion, 8(3), 410.

Grillon, C., Ameli, R., Goddard, A., Woods, S. W., \& Davis, M. (1994). Baseline and fearpotentiated startle in panic disorder patients. Biological Psychiatry, 35(7), 431-439.

Grillon, C., Ameli, R., Merikangas, K. R., Woods, S. W., \& Davis, M. (1993). Measuring the time course of anticipatory anxiety using the fear-potentiated startle reflex. Psychophysiology, 30(4), 340-346.

Grillon, C., Baas, J. M., Pine, D. S., Lissek, S., Lawley, M., Ellis, V., \& Levine, J. (2006). The benzodiazepine alprazolam dissociates contextual fear from cued fear in humans as assessed by fear-potentiated startle. Biological Psychiatry, 60(7), 760-766.

Grillon, C., Baas, J. P., Lissek, S., Smith, K., \& Milstein, J. (2004). Anxious responses to predictable and unpredictable aversive events. Behavioral Neuroscience, 118(5), 916-924.

Grillon, C., Lissek, S., Rabin, S., McDowell, D., Dvir, S., \& Pine, D. S. (2008). Increased anxiety during anticipation of unpredictable but not predictable aversive stimuli as a psychophysiologic marker of panic disorder. American Journal of Psychiatry, 165(7), 898904.

Grillon, C., Pine, D. S., Lissek, S., Rabin, S., Bonne, O., \& Vythilingam, M. (2009). Increased anxiety during anticipation of unpredictable aversive stimuli in posttraumatic stress disorder but not in generalized anxiety disorder. Biological Psychiatry, 66(1), 47-53.

Grupe, D. W., \& Nitschke, J. B. (2013). Uncertainty and anticipation in anxiety: An integrated neurobiological and psychological perspective. Nature Reviews Neuroscience, 14(7), 488501. 
Hsu, M., Bhatt, M., Adolphs, R., Tranel, D., \& Camerer, C. F. (2005). Neural systems responding to degrees of uncertainty in human decision-making. Science (New York, N.Y.), 310(5754), 1680-1683.

Kaye, J. T., Bradford, D. E., \& Curtin, J. J. (2016). Psychometric properties of startle and corrugator response in NPU, affective picture viewing, and resting state tasks. Psychophysiology, 53(8), 1241-1255.

Lissek, S., Baas, J. M., Pine, D. S., Orme, K., Dvir, S., Nugent, M., . . Grillon, C. (2005). Airpuff startle probes: An efficacious and less aversive alternative to white-noise. Biological Psychology, 68(3), 283-297.

Marlin, N. A., Sullivan, J. M., Berk, A. M., \& Miller, R. R. (1979). Preference for information about intensity of signaled tailshock. Learning and Motivation, 10(1), 85-97.

Mineka, S., \& Kihlstrom, J. F. (1978). Unpredictable and uncontrollable events: A new perspective on experimental neurosis. Journal of Abnormal Psychology, 87(2), 256.

Mobbs, D., Petrovic, P., Marchant, J. L., Hassabis, D., Weiskopf, N., Seymour, B., ... Frith, C. D. (2007). When fear is near: Threat imminence elicits prefrontal-periaqueductal gray shifts in humans. Science, 317(5841), 1079-1083.

Nelson, B. D., Hajcak, G., \& Shankman, S. A. (2015). Event-related potentials to acoustic startle probes during the anticipation of predictable and unpredictable threat. Psychophysiology, 52(7), 887-894.

Nelson, B. D., \& Shankman, S. A. (2011). Does intolerance of uncertainty predict anticipatory startle responses to uncertain threat? International Journal of Psychophysiology, 81(2), 107115. 
Sarinopoulos, I., Grupe, D. W., Mackiewicz, K. L., Herrington, J. D., Lor, M., Steege, E. E., \& Nitschke, J. B. (2010). Uncertainty during anticipation modulates neural responses to aversion in human insula and amygdala. Cerebral Cortex, 20(4), 929-940.

Schmitz, A., \& Grillon, C. (2012). Assessing fear and anxiety in humans using the threat of predictable and unpredictable aversive events (the NPU-threat test). Nature Protocols, 7(3), $527-532$.

Shankman, S. A., Robison-Andrew, E. J., Nelson, B. D., Altman, S. E., \& Campbell, M. L. (2011). Effects of predictability of shock timing and intensity on aversive responses. International Journal of Psychophysiology, 80(2), 112-118.

Shankman, S. A., Gorka, S. M., Nelson, B. D., Fitzgerald, D. A., Phan, K. L., \& O'Daly, O. (2014). Anterior insula responds to temporally unpredictable aversiveness: An fMRI study. Neuroreport, 25(8), 596-600.

Williams, L. E., Oler, J. A., Fox, A. S., McFarlin, D. R., Rogers, G. M., Jesson, M. A., . . Kalin, N. H. (2014). Fear of the unknown: Uncertain anticipation reveals amygdala alterations in childhood anxiety disorders. Neuropsychopharmacology, 40(6), 1428-1435. 